\title{
Internet of Nano Things: The World Wide Techno Component Next
}

\author{
M. Prakasam* and M. Jhanani
}

Department of Computer Science - PG, K.S.R College of Arts and Science (Autonomous), Tiruchengode - 637215, Tamil Nadu, India; prakash_mr@rediffmail.com, jhananimsc2013@gmail.com

\begin{abstract}
The combination of nano sensors and nano devices with Internet have form the forum for the development of next modernization regular that deals with various types of data, supports high speed of communication from heterogeneous network namely (Nano link, Nano micro interface and Body Sensor Network, etc) which leads to "Internet of Nano Things" (IoNT). The Internet of Things (IoT) leads future where all the peripherals will be connected via internet and enables their identification as configured devices. This research article clearly explains the role of IoNT in Global Market, details of the Nanotechnology and Nano machines, Network Architecture of IoNT, IoNT applications in various domains and issues of IoNT.
\end{abstract}

Keywords: Body Sensor Networks, Health Monitoring, Internet of Nano Things (IoNT), RFID, Internet of Things (IoT), Nanotechnology, Nanosensors, Nano nodes, Wireless Medical Sensor Network (WMSN)

\section{Introduction}

\subsection{Global Market}

IoNT market has played a vital role in enormous wealth asset for the growth of nanotechnology. IoNT classified its communication type into two types:

1) Electromagnetic Nano-Communication ${ }^{9,15}$ : It is related to the transmission and receiving of electromagnetic (EM) radiation from nanomaterials.

2) Molecular Communication ${ }^{7,8}$ which is concerned with transmission and receiving of encoded information in molecules.

Analyst predicts that the global internet of things (IoT) market will grow at a high Compound Annual Growth Rate of nearly $36 \%$ by 2020 . The cloud based application is widely used by the most of governance digital project in many countries which leads to the implementation of IoT architecture in global market. The prime objective of the IoT implementation in very large scale organization is to increase the business efficiency and process optimization and utilization of the resources. Further, this is contin- ued to manufacture the cost effective smarter devices like media players, imaging devices, map-reading devices etc with the influence of IoT which will significantly increase over the next four years.

An interesting trend in gaining the attraction in this market is the growing demand for big data analytics. The advanced analytical process and knowledge work for extracting the specific information from stored and online data is carried out with help of the specialized tools. The integration of analytics and IoT allows seamless transmission of real-time data between machines and networks, which in turn results in the efficient management of the voluminous data.

The oncoming technology trend will be not on focusing the online computing technology. The Internet of Things (IoT $)^{1,3}$ is nothing but the connectivity of day to day objects with the interconnected networks via internet service. IoT utilized in many domains starting from civil services to defense sectors which includes Healthcare in patient monitoring systems, Agriculture in crop growth monitoring and animal crossing monitoring, space, medicine, protection, construction, water, and Internet of

\footnotetext{
${ }^{*}$ Author for correspondence
} 
Things (IoT) is the enveloping survival of large range of peripherals such as RFID (Radio Frequency Identification) tags, sensors, actuators, mobile technology, NFC (Near Field Communication), Smart Phones, Tablets etc. having exclusive addressing scheme to interrelate and converse among each other to reach desired objectives. IoT concept, nowadays are being a certainty, was projected by Ashton ${ }^{4}$ in 1999 and it is connected to new idea of RFID concerning its usage in deliver sequence. The Internet of Things (IoT) is a scheme of interrelated material and computing devices that are uniquely identifiable and it is capable of transmitting data over a network lacking of human-tohuman or human-to-computer interface in fields such as online health care, online agriculture, online industry, smart cities, e-Defence and so on. Internet of Things of converged with advanced trends like 4G/Lite, GPS, WIFI, PLC, WIFI Max and Big Data Analytics tools, etc. Internet of Things (IoT) requires ${ }^{5}$ shared environment, Intelligence systems, Sensing, Communications Devices and Big Data analytical tools. IoT enables the communication between objects and sensors unlike communication between internet and device. This transformation leads to the invention of new domains like, Wireless Body Sensor Networks (WBAN), Internet of Nanotechnology-Nano Things (IoNT) and everything is leading to one combined terminology i.e. Internet of Everything (IoE) ${ }^{3}$.

Nano machine is the spine of Nanotechnology. A Nanomachine is otherwise known as nanite. Nanite performs the tasks with the help of nano components for collecting the data at high speed which leads to the development of new technology termed as "Internet of Nano-Things (IoNT)" ${ }^{6,7}$.

\section{Nanotechnology and Nanomachines}

\subsection{NanoTechnology}

Nanotechnology refers to make change in the structure of the material on small number of nanometers starting from $0.1 \mathrm{~nm}$ to $100 \mathrm{~nm}$ at atoms or molecular positioning and arrangement. The insight of Nanotechnology was successfully initiated by "Richard Feynman"-A Physicist Nobel Laureate in 1965. According to him, "the scheme must highlight the area of tininess device manufacturing".

The perception of "Nanotechnology" was initially stated by $\mathrm{N}$. Taniguchi $\mathrm{i}^{11}$ as "Nanotechnology generally consist of separating, combining, and warping of materials by means of molecular level or atomic level." The concept of Nanotechnology is trimness as well as an invention of the devices and objects in the extent level of 1 to 100 nano meters.
Passive Nano structure is accomplishing its task by good attribute to change the state during application and or in response to an external control to which it applied. The "Active Nanostructures" are normally used for changing their properties and these active nano structures targeting the drugs and involves in the task like polymer based drug delivery. The next generation of nano technology will support more number of interacting devices on real time applications especially society and corporate.

\subsection{Nanomachines}

Nanomachine is also called as nanite, which consist of mechanical or an electromechanical device. The Nanomachine has the size in terms of nanometers (millionths of a millimeter, or units of $10^{-9}$ meters). Nanomachines are widely used in the scientific research-and-development, but some ancient devices have been tested. It is being utilized both in artificial nanomachines and naturally in the work devices found in biological systems.

The working styles of Nano-Machines have the following approaches. ${ }^{8}$

\subsubsection{Top Down Approach}

It requires a very specific processing of materials with high cost. It is required at when the component size is nano scale dimensions. Example: Nano-electromechanical systems (NEMS).

\subsubsection{Bottom Up Approach}

The nano-machines can be designed with individual molecules. This approach is also known as molecular manufacturing. Example: Nano machines like molecular switches, molecular shuttles etc.

\subsubsection{Bio-Hybrid Approach}

The numbers of nanoscale components which present in the biological cells are considered as nano machines which in turn be used to perform the desired function with the help of set of instruction code written on it. Example: Bio-nano robots, nano-biosensors, biological storing components etc.

\section{Internet of Nano Things (IoNT)}

IoT is working on the concept of identify, sense, Network and process of the ability to optimize the communicating devices and avail services with each other over the internet connectivity to accomplish a specific task. Nanotechnology occupied the vital part of the Internet of Nano Things (IoNT). 
Ian F. Akyildiz and Josep Miguel Jornet from Georgia Institute of Technology was introduced the idea of IoNT. The IoNT is the combination of smaller size network of physical objects which exchanges data together in terms of nano communication.

The architecture which consisting of particularly nano based devices connected with different networks via speedy internet connection leads the new phenomenon known as "Internet of Nano-Things"

Internet of Nano Things (IoNT) communications can be structured by integrating nano devices and a number of other technologies such as IoT, Sensors Network, Cloud Computing, Big Data Analytics etc.

\section{Network Architecture of IoNT}

IoNT practicing the best and new technology based improvement models for data collection, which is a common factor for its successful implementation in real time applications rather than the IoT.

\subsection{Architecture of IoNT}

\subsubsection{Nano-Nodes}

It is the device which is responsible for performing computational task and transmission of data in short distance network with minimal memory. Examples: Bio sensor, DNA Bio sensor, Body Sensor Networks. fitted in Human Body as Nano-Nodes.

\subsubsection{Nano-Routers}

These are having a very large computation capability rather than the nano nodes and acts as device which aggregates the data supplied by nano-nodes. It is greatly controls and maintains the nano nodes.

\subsubsection{Interface devices}

Performs the task of aggregation of information coming from nano-routers and transmit it to the microscale and vice versa. They act as cross devices to converse in nanoscale using Nano communication techniques and also with traditional communication networks with classical network protocols.

\subsubsection{Gateway}

It is responsible for connecting all the nano things available on various networks remotely via internet.

Example:

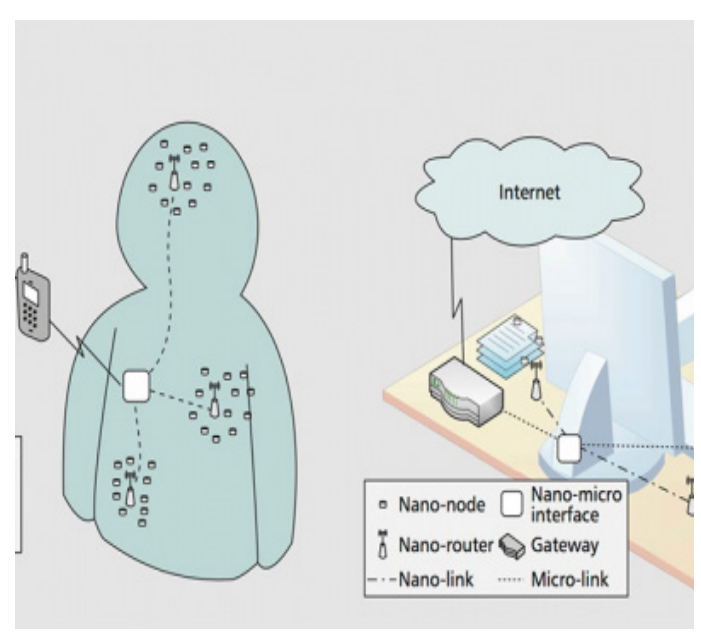

Figure 1. Classical Architecture of IoNT.

The collected data from the Human Body sensor network can be accessed via internet by the authenticated doctors anywhere with the help of smaller handled devices. The gateways are responsible for transferring the data from nano nodes to internet. This communication can be successful by placing the nano micro interface between nano nodes and gateway. The Figure 1 shows the typical Classical Archietecture of IoNT.

\subsection{Nano Based Body Sensor Network ${ }^{6,16}$}

In real world Body Sensor Network (BSN) is the first application where IoNT can be seen which consisting of micro sensors fixed inside the human body completes the job of collecting the bio sensor data, for analyzing and monitoring the activities of molecules and biological activities of the patient. Smarter devices were used for providing alerts and reports about the patient when it is interconnected with the BSN. The Figure 2 shows how the Health Monitoring System is working with the help of IoNT.

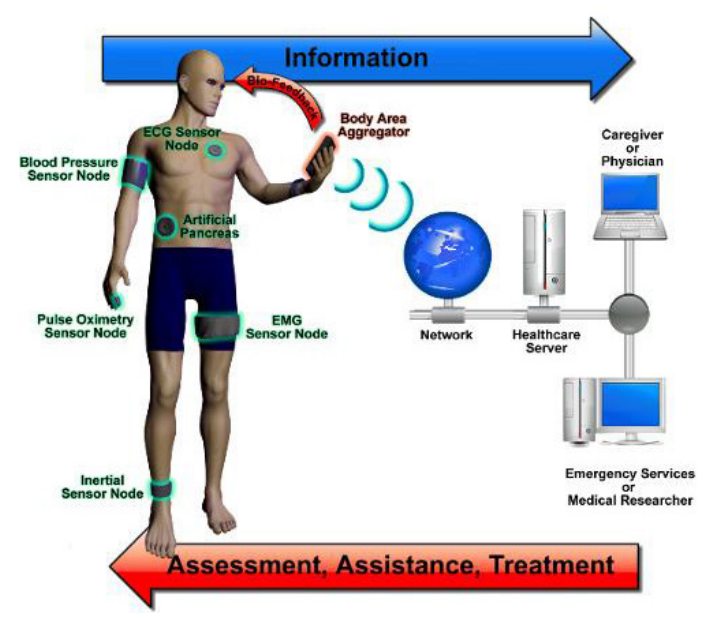

Figure 2. IoNT based Health Monitoring System. 


\subsection{Environmental Monitoring}

IoNT takes care of environmental monitoring systems effectively with the help of Nano sensors in public locations. This environmental monitoring system were successfully implemented in traffic monitoring and atmospheric monitoring domains efficiently

\subsection{Precision Agriculture ${ }^{17}$}

The IoNT implemented successfully in precision farming applications with the help of nano sensors and IoNT networks. This wireless nano sensor network greatly helps for crops growth monitoring and Animal movement monitoring inside the fields.

\subsection{Other Applications}

The technology of IoNT can be further improved and it can be fixed with Army for supporting War field monitoring systems, Manufacturing of Small sized Robotics, Satellite Based communication Applications and in the products of Industry manufacturing invention.

\section{Issues and Challenges of IoNT}

New mechanisms for security and privacy require to be addressed when collecting the data which is done by nano sensors. New improved services and service related architectures have to be proposed to make nano sensors and nano networks well-suited to hold plenty of huge varieties of data. More number of coupled devices results to vulnerabilities and it ends with chance of security threats.

\section{Conclusion and Future Scope}

IoNT have very significant impact on many fields from Health care to environmental monitoring, precession agriculture, War filed monitoring and Nano robotics, etc. Further it can be used wide spread in near future. In this paper a detailed review process is carried out in regards with IoNT for better understanding which initiates new innovative ideas for doing research. In future IoNT based sensors and connective devices and networks can be utilized for most of the monitoring systems in different arena. This will serve a better platform for the young researchers and budding nano scientist.

\section{References}

1. Xia F, Yang LT, Wang L, Vinel A. Internet of things. International Journal of Communication Systems. 2012; 25(9):1101. https:// doi.org/10.1002/dac. 2417
2. Nayyar A, Puri V, Le D-N. Internet of Nano Things (IoNT): Next Evolutionary Step in Nanotechnology

3. Atzori L, Iera A, Morabito G. The internet of things: A survey Computer networks. 2010; 54(15):2787-805. https://doi. org/10.1016/j.comnet.2010.05.010

4. Ashton K. That 'Internet of Things' thing. RFID Journal. 2009.

5. Gubbi J, Buyya R, Marusic S, Palaniswami M. Internet of Things (IoT): A vision, architectural elements, and future directions. Future Generation Computer Systems. 2013; 29(7):1645-60. https://doi.org/10.1016/j.future.2013.01.010

6. Balasubramaniam S, Kangasharju J. Realizing the internet of nano things: challenges, solutions, and applications. Computer. 2013; 46(2):62-8. https://doi.org/10.1109/ MC.2012.389

7. Akyildiz IF, Jornet JM. The Internet of Nano-Things. IEEE Wireless Communications. 2010; 17(6):58-63. https://doi. org/10.1109/MWC.2010.5675779

8. Akyildiz IF, Brunetti F, Blazquez C. Nanonetworks: A New Communication Paradigm. Computer Networks (Elsevier) J. 2008 Aug; 52(12):2260-79.

9. Akyildiz IF, Jornet JM. Electromagnetic Wireless Nanosensor Networks. Nano Communication Networks (Elsevier) J. 2010 Mar; 1(1):3-19.

10. Feynman RP. There's plenty of room at the bottom. Miniaturization. (HD Gilbert, ed.) Reinhold, New York. 1961.

11. Taniguchi N. On the basic concept of nano-technology. In Proceeding of the International Conference on Production Engineering. 1974. PMCid:PMC2139507

12. Roco MC, Mirkin CA, Hersam MC. Nanotechnology research directions for societal needs in 2020: retrospective and outlook. Springer Science \& Business Media. 2011; 1. https://doi. org/10.1007/978-94-007-1168-6, https://doi.org/10.1007/97894-007-1168-6_1

13. Suda T, Moore M, Nakano T, Egashira R, Enomoto A. Exploratory research on molecular communication between nanomachines. Proceedings of Genetic and Evolutionary Computation Conference (GECCO'05). 2005 Jun.

14. Drexler E. Nanosystems: Molecular Machinery, Manufacturing, and Computation. John Wiley and Sons Inc. 1992.

15. Rutherglen C, Burke P. Nanoelectromagnetics: Circuit and Electromagnetic Properties of Carbon Nanotubes. Small. 2009 Apr; 5(8):884-906. https://doi.org/10.1002/smll.200800527 PMid:19358165

16. Jarmakiewicz J, Parobczak K. On the Internet of Nano Things in healthcare network. International Conference on Military Communications and Information Systems. 2016 May. p. 1-6. https://doi.org/10.1109/ICMCIS.2016.7496572

17. Bhargava K, Ivanov S, Donnelly W. Internet of Nano Things for Dairy Farming. In Proceedings of the Second Annual International Conference on Nanoscale. Computing and Communication. 2015 Sep. p. 24. https://doi. org/10.1145/2800795.2800830 